\section{Proof of Concept for Robot-aided Upper Limb Rehabilitation Using Disturbance Observers}

\author{
Barkan Ugurlu, Member, IEEE, \\ Masayoshi Nishimura, Kazuyuki Hyodo, Member, IEEE, \\ Michihiro Kawanishi, Member, IEEE, and Tatsuo Narikiyo
}

\begin{abstract}
This paper presents a wearable upper body exoskeleton system with a model based compensation control framework to support robot-aided shoulder-elbow rehabilitation and power assistance tasks. To eliminate the need for EMG and force sensors, we exploit off-the-shelf compensation techniques developed for robot manipulators. Thus target rehabilitation tasks are addressed by using only encoder readings.

A proof of concept evaluation was conducted with 5 able-bodied participants. The patient-active rehabilitation task was realized via observer-based user torque estimation, in which resistive forces were adjusted using virtual impedance. In the patient-passive rehabilitation task, the proposed controller enabled precise joint tracking with a maximum positioning error of 0.25 degrees. In the power assistance task, the users' muscular activities were reduced up to $85 \%$ while exercising with a $5[\mathrm{~kg}]$ dumbbell. Therefore, the exoskeleton system was regarded as being useful for the target tasks; indicating that it has a potential to promote robot-aided therapy protocols.
\end{abstract}

Index Terms-exoskeleton, robot-aided rehabilitation, power assistance, disturbance observer, user torque estimation.

\section{INTRODUCTION}

Robotic techniques in rehabilitation and power assistance tasks may increase operational efficiency in enabling patients to recover from acute and chronic stroke [1]-[3]. Medical personnel may also benefit from the use of such equipment while performing nursing care, e.g., moving or lifting relatively heavy objects in a daily routine. A multipurpose exoskeleton may be a useful asset for robot-assisted rehabilitation and power assistance tasks. Therefore, this research focuses on the development of a wearable and multitasking upper limb exoskeleton.

With respect to upper limb rehabilitation devices, Perry et al. developed an upper body exoskeleton, actuated via cable transmission systems [4]. Nef et al. engineered ARMin III, an active exoskeleton that is modeled on the basis of the human shoulder actuation principle [5]. For robot-aided neurorehabilitation, Krebs et al. built an impedance-controlled mechanism called MIT-MANUS [6]. Tsagarakis and Caldwell constructed a 2-D haptic assistive system to minimize pathological absence of motor control in upper limb impaired patients [7]. Sanchez et al. introduced T-WREX, a 5-DoF mechanism that passively counterbalances the patient's arm weight using elastic bands [2]. Rosati and his colleagues developed NeReBot, a wire-length-controlled robot for post-stroke rehabilitation [8]. Mao and Agrawal introduced a cable-driven tendon system that

Manuscript received December 13, 2013; revised April 14, 2014, June 10, 2014, July 22, 2014. Accepted October 2, 2014.

This paper has supplementary downloadable material available at http://ieeexplore.ieee.org, provided by the authors. This includes a multimedia MPEG4 format movie clip, which show scenes from experiments. This material is $36.2 \mathrm{MB}$ in size.

B. Ugurlu is with the Dept. of Mechanical Engineering, Ozyegin University, Istanbul 34794, Turkey. He is also with the Computational Neuroscience Laboroties, ATR, 619-0288 Kyoto, Japan. (e-mail: barkanu@ieee.org).

M. Nishimura was with the the Dept. of Advanced Science and Technology, Control Systems Lab., Toyota Technological Institute, Nagoya 468-8511, Japan. He is now with Suzuki Motor Corporation, Hamamatsu 432-8611, Japan. (e-mail: nishimuram@hhq.suzuki.co.jp).

M. Kawanishi and T. Narikiyo are with the Dept. of Advanced Science and Technology, Control Systems Lab., Toyota Technological Institute, Nagoya 468-8511, Japan. (e-mail: kawa@toyota-ti.ac.jp; n-tatsuo@toyota-ti.ac.jp).

$\mathrm{K}$. Hyodo is with the Dept. of Computer Science and Engineering, Fukuoka Institute of Technology, Fukuoka 811-0295, Japan. (e-mail: hyodo@fit.ac.jp). can generate push-pull forces [9]. The majority of these systems are immobile as they are designed for specific rehabilitation tasks rather than possessing the multitasking functionality.

In [10] and [11], Ohnishi et al. described the disturbance observer (DOB) which makes use of the inverse plant model to estimate the resultant disturbance acting on the system. DOB modules improve robustness and tracking accuracy of industrial manipulators. They are also used in haptics and power assistance control [12], [13]. We defend that their usage in exoskeleton systems could be generalized for estimating user-applied force variations if other disturbances caused by robot dynamics and joint friction are sufficiently compensated.

This work has two main objectives:

1) Competency via DOB-based User Torque Estimation: In our setup, disturbance observer units estimate the task-specific interaction forces, by virtue of the proposed compensation schemes. In realizing the target rehabilitation tasks via DOB-based force estimation, the device should show similar performance to other systems with force sensors.

2) Reduced Complexity and Multitasking: We design the system to reduce manufacturing and maintenance costs through the use of commercially available components. Power assistance and rehabilitation functions are activated without any requirement for EMG modules, expensive force sensors or joint-level torque sensing units. While pursuing a sensorless approach, a light, wearable, and mobile multipurpose exoskeleton is designed for specific target applications.

We have composed a compensation controller framework via the combination of off-the-shelf techniques developed for robot manipulators (full dynamics compensation, friction compensation, disturbance observer, virtual impedance, force estimation). Despite potential limitations due to modeling uncertainties, we push the limits of the "encoder only" approach, so as to reduce mechatronic hardware complexity.

This work extends [14] with proof of concept evaluations. The rest of the paper includes a description of the wearable exoskeleton system, details regarding the implementation of rehabilitation tasks, and discussions on the concept evaluation.

\section{The Wearable Exoskeleton System}

For robotic rehabilitation and power assistance implementations in active orthoses, we developed a whole-body active exoskeleton system, named TTI-Exo (TTI stands for Toyota Technological Institute), see Fig. 1. The system includes both lower and upper extremities to deliver overall power augmentation to the human-wearer. Here, we investigate the upper extremity of the exoskeleton which includes 6 DoFs. Each arm includes 2 active joints for the shoulder and elbow rotating along the flexion/extension axis, and 1 passive joint for the shoulder rotating along adduction/abduction axis (Fig. 2).

To reduce the overall weight, links are made of laser molded nylon-resin composite material, which is fairly light but sufficiently rigid. Link lengths of the exoskeleton system are adjustable for various human wearers and human-robot connections are provided by means of straps and belts. Maximal link lengths and general mechanical specifications are in Table I. To actuate the exoskeleton, Harmonic Drive FHA-14C-Mini series of brushless servo motors are employed (http://www.harmonicdrive.net/). These modules include integrated encoders and comparatively light harmonic gears with partial backdrivability ${ }^{1}$. They can be driven via torque input commands by virtue of the inner torque control loop, which additionally handles mechanical transmission losses. However, they are not able to monitor motor current or torque output. See Table II for the actuator specifications.

${ }^{1}$ Note that robot joints become completely backdrivable when friction compensation is applied [29]. See subsection III-D. 


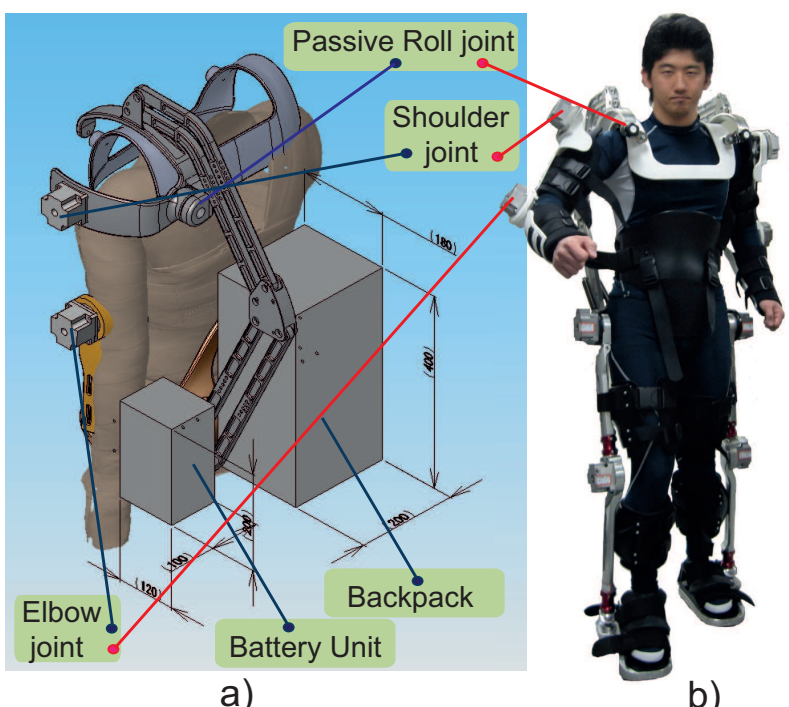

a) b)
TABLE I

TTI-EXo MECHANICAL SPECIFICATIONS (UPPER BoDy)

\begin{tabular}{|c|c|}
\hline Waist-Shoulder $\left(p_{z}\right)$ & $45[\mathrm{~cm}]$ \\
\hline Shoulder to Shoulder $\left(2 p_{y}\right)$ & $40[\mathrm{~cm}]$ \\
\hline Shoulder-Elbow $\left(L_{1}\right)$ & $28[\mathrm{~cm}]$ \\
\hline Elbow-Wrist $\left(L_{2}\right)$ & $27.5[\mathrm{~cm}]$ \\
\hline Max. Static Payload $($ Cont.) & $5[\mathrm{~kg}] \times 2$ \\
\hline Single Arm Weight & $2.8[\mathrm{~kg}]$ \\
\hline Back Unit Weight & $6.4[\mathrm{~kg}]$ \\
\hline Shoulder Joint Range & $-10 \sim 115[\mathrm{Deg}]$ \\
\hline Elbow Joint Range & $5 \sim 120[\mathrm{Deg}]$ \\
\hline
\end{tabular}

TABLE II

ACTUATOR SPECIFICATIONS: FHA-14C MiNI

\begin{tabular}{|c|c|}
\hline Max. Torque (Cont.) & $28[\mathrm{Nm}]$ \\
\hline Max. Torque (Peak) & $75[\mathrm{Nm}]$ \\
\hline Max. Velocity & $2 \pi[\mathrm{rad} / \mathrm{s}]$ \\
\hline Encoder Res. (Inc.) & $800000[\mathrm{pulses} / \mathrm{rev}]$. \\
\hline Mass & $1.2[\mathrm{~kg}]$ \\
\hline Gear Ratio & 100 \\
\hline
\end{tabular}

Fig. 1. a) CAD drawing of the upper extremity exoskeleton system. b) The actual whole-body exoskeleton.

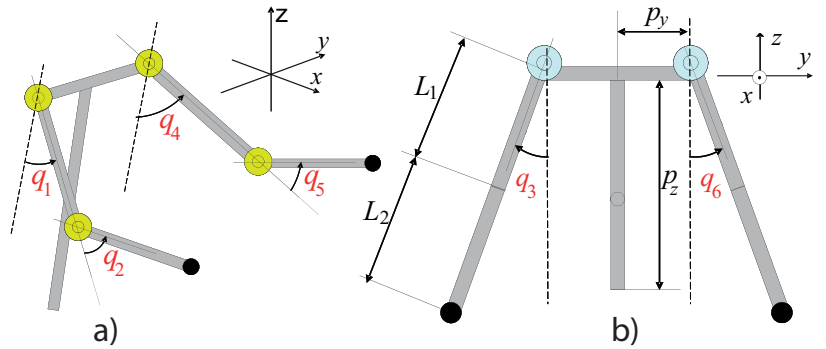

Fig. 2. a) Joint configurations are displayed in an isometric perspective. b) Joint configurations in the lateral plane. $\left\{q_{1}, q_{2}\right\}$ and $\left\{q_{4}, q_{5}\right\}$ are right and left arm's active shoulder and elbow joints, respectively. $q_{3}$ and $q_{6}$ are passive roll joints in right and left arms. Link information is given in Table I.

The main controller is an Intel TMD Duo $2 \mathrm{CPU} 3.33 \mathrm{GHz}$ processor with 4.0 GB RAM, running on a 32-bit Windows operating system that includes MATLAB xPC Target. It is capable of realizing all the tasks in real-time with a sampling time of 1 [ms]. The main controller unit, motor drives and other electronic peripherals are highly integrated in a backpack unit (Fig. 1(a)). The system is powered via dual Li-Ion batteries, on both sides of the backpack. The whole system is wearable and autonomous.

\section{COMPEnSATion Schemes}

When the exoskeleton is in motion, it is subject to forces that are based on its dynamics; namely, gravitational forces, inertial forces, coriolis and centrifugal forces. Using manipulator dynamics, we can compute the necessary feedforward joint torques to compensate for the effects of these forces. In addition to those, stiction and viscous friction are inevitable in exoskeleton joints. To address such disturbances, friction identification procedures and friction compensation algorithms are implemented. Having identified and compensated disturbances based on robot dynamics and joint friction, we use disturbance observers to estimate external torques acting on the system. In other words, model-based compensation of the forces that are based on dynamics and joint-level disturbances is essential in our approach.

\section{A. Robot Dynamics Model}

Robot dynamics for a torque-controlled single TTI-Exo arm is as follows,

$$
\begin{aligned}
& M(\theta) \ddot{\theta}+C(\theta, \dot{\theta}) \dot{\theta}+G(\theta)=T_{l}+T_{e x t}, \\
& T_{c m d}=J_{m} \ddot{\theta}+F_{r}(\dot{\theta})+T_{l},
\end{aligned}
$$

where $\theta$ is the joint position vector, $T_{l}$ is the joint torque vector at the link side, $J_{m}$ is a diagonal matrix that stores actuator rotor inertia values with appropriate scaling via respective gear ratios. $T_{e x t}$ is the vector of external torques acting on the system. $F_{r}(\dot{\theta})$ symbolizes overall joint friction terms and we analyze it in subsection III-D. $T_{c m d}$ is the final motor torque command and it can be re-assessed by combining (1) and (2) to eliminate $T_{l}$.

$$
T_{c m d}=M(\theta) \ddot{\theta}+C(\theta, \dot{\theta}) \dot{\theta}+G(\theta)+J_{m} \ddot{\theta}+F_{r}(\dot{\theta})-T_{e x t}
$$

$M(\theta), G(\theta), C(\theta, \dot{\theta})$ are inertia, gravity, coriolis and centrifugal terms which can be computed by using CAD data. Elements of $J_{m}$ are acquired by actuator manufacturer data and double-checked using identification procedures. Counter-electromotive force is compensated in the motor drives by default, thus, not considered in (2).

\section{B. Overall Compensation Implementation}

In order to cancel the forces that are based on robot dynamics and friction, we can compute the associated joint compensation torques and implement them as feedforward inputs to TTI-Exo's torque-controlled actuators (Fig. 3). $T_{i n p}, f$ and $s$ respectively symbolize the task-specific reference torque input, low pass filter frequency and Laplace domain variable. $T_{f r}$ is the friction compensation torque which corresponds to the $F_{r}(\dot{\theta})$ term and it will be covered in subsection III-D. Furthermore, $T_{m}, T_{g r}$, and $T_{c c}$, stand for compensation torques that are associated with inertia, gravity, coriolis and centrifugal terms. Observing Fig. 3, $T_{c m d}$ is chosen as $T_{c m d}=T_{i n p}+T_{m}+T_{c c}+T_{g r}+T_{f r}$. Therefore, (3) takes the following form.

$$
\begin{aligned}
& T_{i n p}+T_{m}+T_{c c}+T_{g r}+T_{f r}=M(\theta) \ddot{\theta}+C(\theta, \dot{\theta}) \dot{\theta} \\
& +G(\theta)+J_{m} \ddot{\theta}+F_{r}(\dot{\theta})-T_{e x t}
\end{aligned}
$$




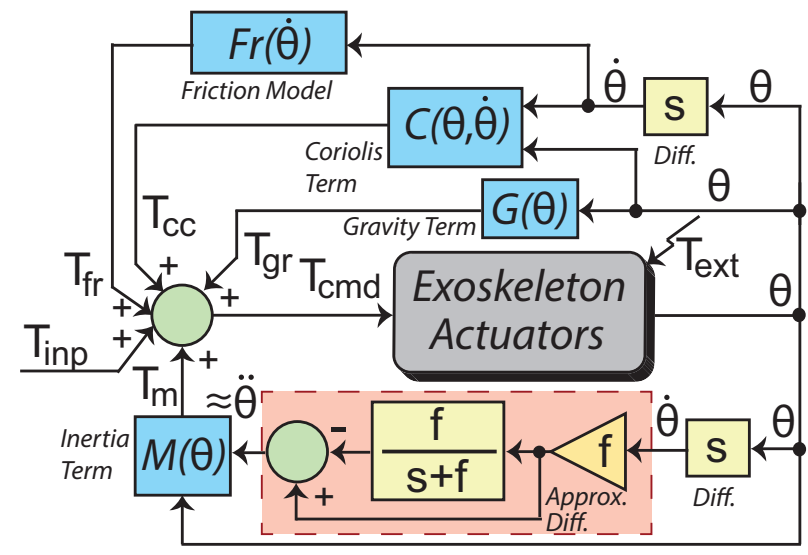

Fig. 3. Overall compensation scheme. All frictional, inertial, coriolis and gravitational forces are compensated. We utilized an approximate differentiation block while obtaining joint acceleration.

Assuming that the robot model and friction parameters are sufficiently precise, $T_{m}, T_{c c}, T_{g r}$ and $T_{f r}$ respectively cancel out the $M(\theta) \ddot{\theta}, C(\theta, \dot{\theta}) \dot{\theta}, G(\theta)$ and $F_{r}(\dot{\theta})$ terms. Finally, the multi-DoF robot dynamics could be reduced to the decoupled equation expressed below.

$$
T_{i n p}+T_{e x t}=J_{m} \ddot{\theta}
$$

While implementing the scheme in Fig. 3, joint velocities are low-pass filtered with a frequency of 32 [Hz]. In the approximate differentiation block for joint acceleration, the low pass frequency is chosen as $4.8[\mathrm{~Hz}]$, as it is more prone to noise. These frequencies are empirically determined to address the trade-off between the delay and output noise cancellation.

\section{Disturbance Observer}

A basic DOB scheme for a general $i^{\text {th }}$ joint is illustrated in Fig. 4 [10]. $T_{u i}, P_{i}(s), T_{d i}, \hat{T}_{d i}$ and $J_{m i}$ respectively stand for input torque, motor plant model, disturbance torque, estimated disturbance torque and rotor inertia. The basic principle behind DOB is to invert the plant model and obtain an approximation for $T_{d i}$, as follows.

$$
\begin{aligned}
& \hat{T}_{d i}=f J_{m i} s \theta_{i}-\frac{f}{s+f}\left(T_{u i}+f J_{m i} s \theta_{i}\right) \\
& \hat{T}_{d i}=\frac{s^{2} J_{m i} \theta_{i}}{\frac{1}{f} s+1}-\frac{1}{\frac{1}{f} s+1} T_{u i} \\
& \hat{T}_{d i} \cong\left(J_{m i} \ddot{\theta}_{i}-T_{u i}=T_{d i}\right)
\end{aligned}
$$

The first term in (7) includes an ideal derivative and an approximate derivative with a time constant of $\frac{1}{f}$. Double differentiating the $J_{m i} \theta_{i}$ value, and subtracting the low pass filtered $T_{u i}$, we can obtain $\hat{T}_{d i}$. For details, refer to [10].

The output of DOB $\left(\hat{T}_{d i}\right)$ should be fed back only for position control schemes. In doing so, DOB rejects disturbances based on parameter variations and dynamic loads; enhancing the joint-tracking performance and therefore the joint stiffness. For force control schemes, it should not be fed back. Otherwise, the human-wearer cannot move his arm since DOB senses human movement as a disturbance force and rejects it. Therefore, DOB output can be utilized for force control schemes as a tool to estimate the external torques acting on the system, as long as other disturbance effects (dynamics load, stiction and friction) are sufficiently compensated.

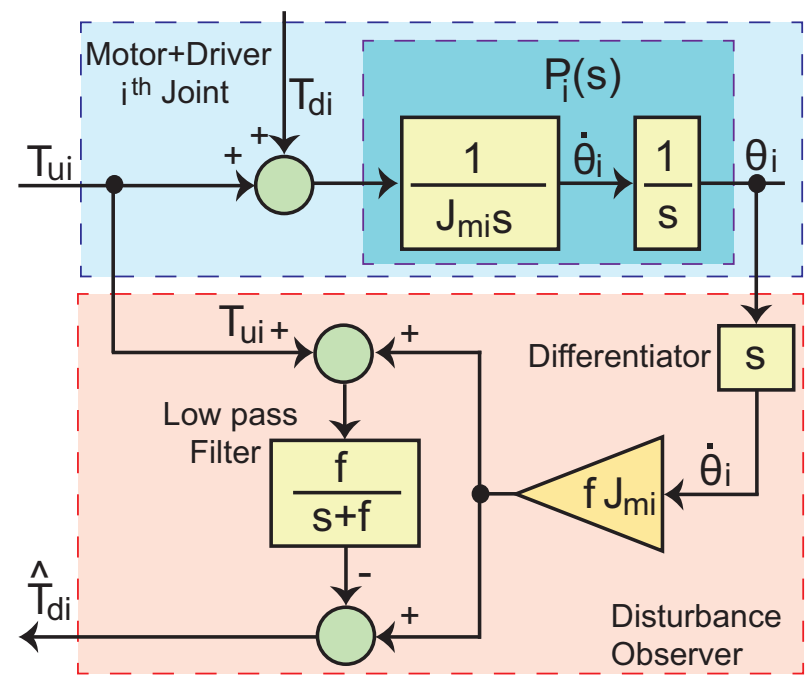

Fig. 4. Basic disturbance observer scheme.

\section{Friction Compensation}

With a priori full dynamics compensation, stiction and viscous friction appear to be the remaining dominant disturbance characteristics in any joint module. To identify stiction and viscous friction parameters, we conduct an identification routine in which the joints are individually driven via a ramp input [11].

In Fig. 5, we observe frictional disturbance torque - angular velocity curves for shoulder and elbow joints. Solid purple (dark) lines indicate the experimentally collected data while solid cyan (light) lines point out the identified stiction and viscous friction models obtained through curve fitting. Identified stiction and viscous friction models for the shoulder and elbow joints can mathematically be expressed as follows; $F_{r}(\dot{\theta})=T_{f r}=\left[T_{f r s} T_{f r e}\right]^{T}$.

$$
\begin{gathered}
T_{\text {frs }}=\mu_{1}\left(0.368+0.067 \dot{\theta}_{1}\right)-\mu_{2}\left(0.236-0.056 \dot{\theta}_{1}\right) ; \\
T_{\text {fre }}=\mu_{3}\left(0.355+0.078 \dot{\theta}_{2}\right)-\mu_{4}\left(0.225-0.068 \dot{\theta}_{2}\right) .
\end{gathered}
$$

The joints possess distinct friction characteristics depending on the turning direction. Additional coefficients $\left(\mu_{1}, \mu_{2}, \mu_{3}, \mu_{4}\right)$ are introduced in (9) and (10) as follows.

$$
\begin{aligned}
& \mu_{1}=0.5 \lambda_{1}\left(1+\operatorname{sgn}\left(\dot{\theta}_{1}\right)\right) ; \mu_{2}=0.5 \lambda_{1}\left(1-\operatorname{sgn}\left(\dot{\theta}_{1}\right)\right) ; \\
& \mu_{3}=0.5 \lambda_{2}\left(1+\operatorname{sgn}\left(\dot{\theta}_{2}\right)\right) ; \mu_{4}=0.5 \lambda_{2}\left(1-\operatorname{sgn}\left(\dot{\theta}_{2}\right)\right) .
\end{aligned}
$$

Considering (11) and (12); if ( $\left.\dot{\theta}_{1}>0\right), \mu_{1}=1$ and $\mu_{2}=0$. When $\left(\dot{\theta}_{1}<0\right), \mu_{1}=0$ and $\mu_{2}=1$. Likewise; if $\left(\dot{\theta}_{2}>0\right), \mu_{3}=1$ and $\mu_{4}=0$. When $\left(\dot{\theta}_{2}<0\right), \mu_{3}=0$ and $\mu_{4}=1$. Moreover $\lambda_{1}=\left|\operatorname{sgn}\left(\dot{\theta}_{1}\right)\right|$ and $\lambda_{2}=\left|\operatorname{sgn}\left(\dot{\theta}_{2}\right)\right|$. Therefore, when $\dot{\theta}_{1}=0$, $\left\{\mu_{1}, \mu_{2}\right\}=\{0,0\}$ and when $\dot{\theta}_{2}=0,\left\{\mu_{3}, \mu_{4}\right\}=\{0,0\}$.

The joints become highly backdrivable with the friction compensation scheme [29]. To graphically observe this feature, joint friction parameters are re-identified after implementing friction compensation in accordance with (9) and (10). Fig. 6 shows results for the shoulder and elbow joints, where the remaining frictional characteristics appear to be very small and negligible. Even though it is theoretically possible to conduct a complete compensation, we choose to leave $0.01[\mathrm{Nm}]$ of stiction and $0.025[\mathrm{Nms} / \mathrm{rad}]$ of viscous friction due to: i) complete friction compensation may lead to over compensation, which then might cause unexpected joint motions, and ii) in implementing inertia compensation using approximate differentiators, a damping term must be left to avoid possible instability issues [15]. 

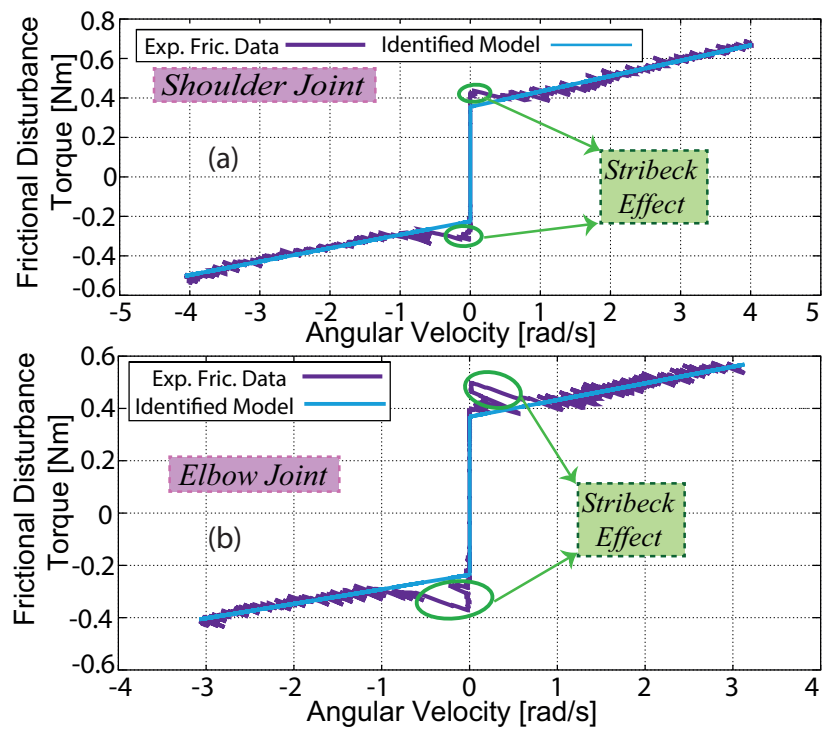

Fig. 5. Stiction and viscous friction identification plots.

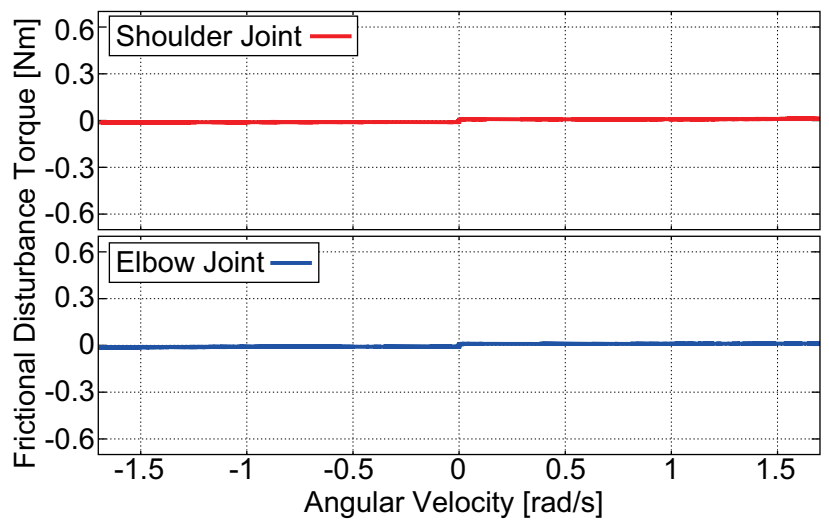

Fig. 6. Remaining frictional characteristics after implementing friction compensation.

Refer to [29] to view scenes from hardware experiments with and without friction compensation.

\section{Methods}

\section{A. Participants}

Five able-bodied volunteers ( 3 male) aged 23 to 59 participated in the evaluation. The internal ethics review board of Toyota Technological Institute approved the study.

\section{B. Target Tasks}

This study specifically targets threee robot-aided tasks.

1) Patient-Active Rehabilitation: In this therapy protocol, the rehabilitation device applies adjustable resistive forces against the patient movement to enable the patient to regain muscle strength in the process of time [16].

For this task, a software-controlled virtual spring damper model is introduced to each joint. The initial joint position corresponds to the spring position at rest. As the patient exerts a force to the system, the joints move but apply resistive force to turn back to their initial position. Depending on the selection of spring damper coefficients $\left(k_{i}, b_{i}\right)$, the resistive force profile can be adjusted. It is also possible to create a medium with pure damping. This approach has been used

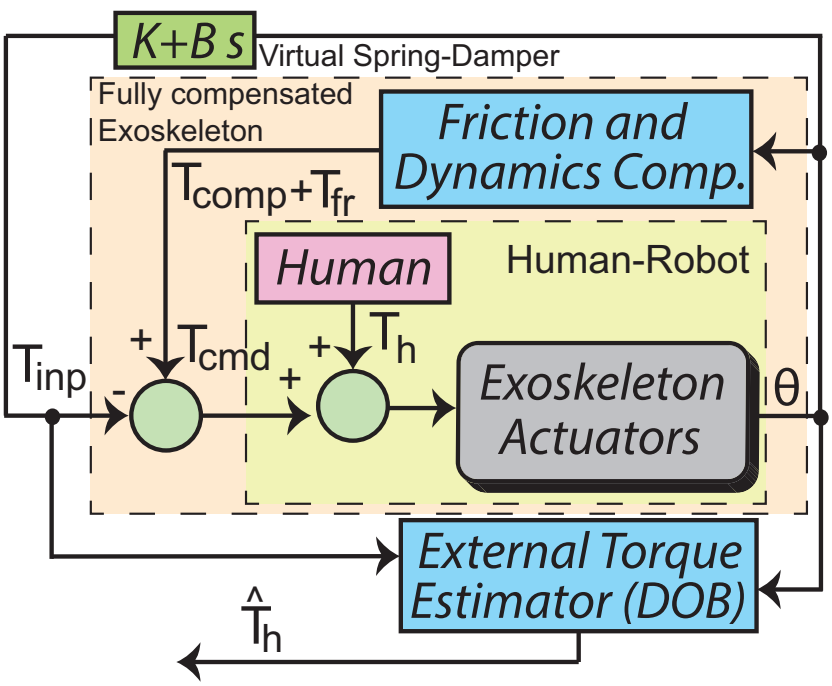

Fig. 7. Active compliance control mode for patient-active rehabilitation task. Friction and Dynamics Comp. block stands for the overall compensation scheme that is illustrated in Fig. 3; $T_{c o m p}=T_{g r}+T_{c c}+T_{m}$.

in [6], [17], [18]. To evaluate muscle strengthening rehabilitation performance, without using any force, torque or EMG sensors, we enhance this approach via the estimation of human-wearer torque.

We implemented the impedance control scheme in Fig. 7. Jointlevel disturbances that are based on frictional and dynamic loads are compensated in accordance with the methods described in section III. If the only external force is the human-applied force $\left(T_{h}\right)$, e.g., nobody pushes the exoskeleton, $T_{\text {ext }}$ corresponds to $T_{h}$. The compensated robot dynamics in this case is expressed as follows.

$$
\begin{aligned}
& T_{i n p}+T_{h}=\{-K \theta-B \dot{\theta}\}+T_{h}=J_{m} \ddot{\theta} \\
& T_{h}=J_{m} \ddot{\theta}+\{K \theta+B \dot{\theta}\}=J_{m} \ddot{\theta}-T_{i n p}
\end{aligned}
$$

Here, $K$ and $B$ are diagonal matrices whose elements are virtual spring damper coefficients $\left(k_{i}, b_{i}\right)$. Moreover, the virtual impedance is implemented via $T_{i n p}$, the task-specific input torque. (14) is identical to (8). As the system is hypothetically free from all the disturbances and dynamic couplings, DOB units placed in each joint supports estimating the external torques exerted by the human-wearer joints, without any force sensing unit.

2) Patient-Passive Rehabilitation: The patient-passive rehabilitation task entails periodically moving the patient's joints via the exoskeleton, in which the patient is not able to perform voluntary muscular activities. It is conducted to prevent rhabdomyolysis, an endemic muscle disease in stroke patients [19].

In this therapy protocol, the system is run in the position control mode, usually with low joint velocity profiles which are required for this rehabilitation task. In the conventional approach, a high gain PID controller is implemented. There are three main challenges which may not be solely handled by high gain PID controllers.

i) Human arm inertial parameter variation: In this therapy mode, the patient cannot exert forces to carry his/her own arm; therefore, the robot must sustain the arm weight. Depending on the human-wearer's arm weight, the controller performance might change. Therefore, instead of fine-tuning PID controller parameters for each person, we utilize DOB feedback so as to eliminate disturbances based on human arm inertial parameter variations [10].

ii) Joint friction: In motion profiles with low joint velocities, stiction and viscous friction have an effect on the robot motion. 


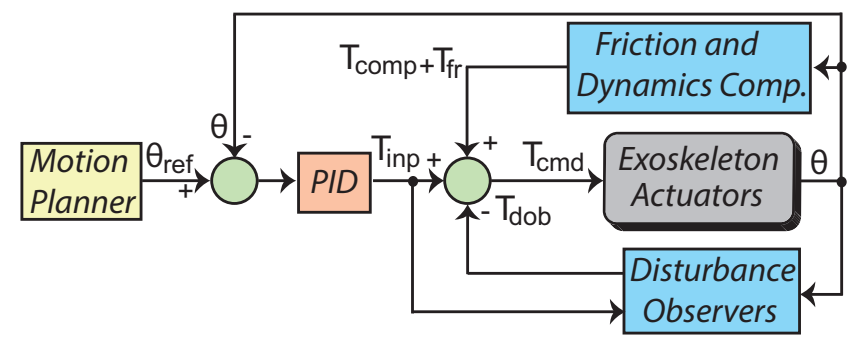

Fig. 8. Position control mode for patient-passive rehabilitation task. Friction and Dynamics Comp. block stands for the overall compensation scheme that is illustrated in Fig. 3; $T_{\text {comp }}=T_{g r}+T_{c c}+T_{m}$.

A high gain PID controller may not reject disturbances based on frictional characteristics, as they could be out of the controller bandwidth in such low-velocity motions. Hence, this problem can be solved via friction compensation schemes [14].

iii) Joint tracking with lower PID gains: In addition to friction compensation, we implement gravity, coriolis and inertial force compensation. Therefore, the position controller performance can be enhanced and we attain satisfactory joint-tracking with comparatively lower gains.

Fig. 8 illustrates the implemented position control scheme for this task, in which the motion planner generates the reference joint angle trajectories. Subsequently, the PID controller computes the necessary torque input to realize the reference joint positions. To handle disturbances based on friction and dynamics loads, friction and dynamics compensation schemes are implemented. In addition, DOB is fed back to reject disturbances based on human-wearer parameter variations and other unmodeled factors.

3) Power Assistance: With assistive mechanical power to the human-wearer, the user can carry or manipulate relatively heavy objects which may reduce fatigue, back pains and related injuries. While providing the assistive mechanical power, the main objective is to follow the human-wearer's motion without causing any limitation and to augment power when necessary. Therefore, the system runs using torque control. The tip weight to be manipulated can be included in the robot motion equations to compensate its dynamic load effects, especially the gravitational load. Friction compensation is also essential and thus we implement the compensation strategy shown in Fig. 3, while assessing the tip weight in the system parameters, i.e., robot dynamics with a load at the end-effector.

\section{Procedures}

For each task, different procedures were implemented. Participants were briefed before starting.

Regarding the patient-active rehabilitation protocol, participants moved their arms while wearing the impedance-controlled exoskeleton. Each session was conducted twice; with 'stiff' and 'soft' spring configurations. Participants were asked to move their arms identically in both cases. Although the degree of 'stiff' and 'soft' were adjusted in accordance with each participant's own preferences, it is possible to set certain impedance values as well. Damping coefficients were kept relatively low but not zeroed to avoid instability.

Concerning the patient-passive rehabilitation protocol, we conducted tests in which joints were driven via a sine wave signal with 5 [Deg] amplitude and $0.3[\mathrm{~Hz}]$ frequency. The PID controller gains were tuned in accordance with the actuator dynamics and not changed during the evaluation. To validate the efficiency of this approach, this test was conducted twice: i) using the 'proposed' scheme in Fig. 8, ii) using the conventional high gain 'PID' control with no compensation and with no DOB feedback.

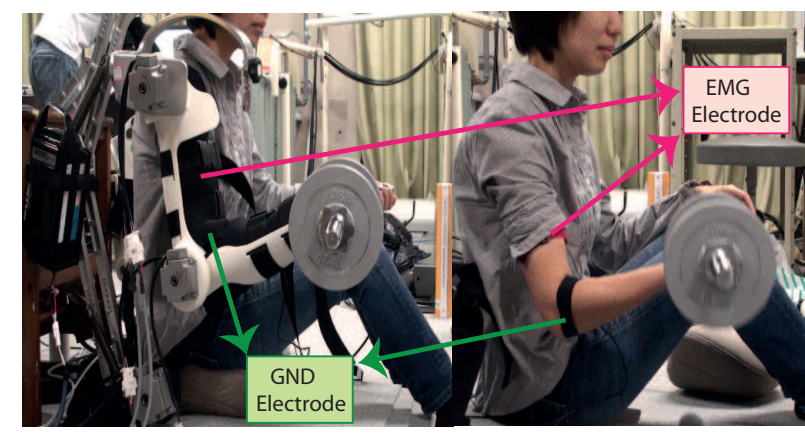

Fig. 9. Participant \#5, while performing $5[\mathrm{~kg}]$ dumbbell lifting exercise for demonstrating the power assistance task [29]. Left hand side, she wears the upper extremity exoskeleton. Right hand side, she performs the same exercise without wearing the exoskeleton arm.

For the power assistance task, five participants lifted dumbbells in three cases: 1) without wearing the exoskeleton system, 2) while wearing the exoskeleton with only gravity compensation for the robot and the tip weight (baseline algorithm), and 3) while wearing the exoskeleton with the full compensation functionality. In the literature, this task is usually implemented by solely considering the gravitational loads. Therefore, power assistance with only gravity compensation for the robot and the tip weight (Case 2) was considered as the baseline algorithm to perform benchmarking. As a tip weight, we chose a $5[\mathrm{~kg}]$ dumbbell.

\section{Data Collection}

In patient-active rehabilitation and power assistance evaluations, the participants moved their shoulder and elbow joints along the flexion/extension axis to create vertical 'up-and-down' motion (see Fig. 9). EMG data were collected from participants' biceps brachii muscles, the dominant skeletal muscles while moving shoulder and elbow joints in the flexion/extension direction [20], [21]. GND (Ground) signal was acquired from a bone in the elbow joint.

While collecting EMG data, we used a dual channel wireless EMG sensor with non-invasive electrodes. Acquired signals were filtered through a band-pass filter within the frequency range of 20-450 $[\mathrm{Hz}]$. DC offset and trends were removed and the resulting signal was fully rectified for further processing. A digital non-casual FIR linear phase low-pass filter extracted the envelope of the signal, which approximately corresponded to the muscular activity. EMG signals were normalized to the maximum, via voluntary muscular contraction by the participants [22]. EMG signals were collected for performance evaluation in patient-active rehabilitation and power assistance evaluations. Moreover, a metronome was used to guide the human-wearer to perform tasks in a periodical manner. In patientpassive rehabilitation evaluation, actual joint angle measurements were collected using the built-in encoders.

\section{RESUlTS}

\section{A. Patient Active Rehabilitation Results}

Results appear in Fig. 10 where the solid green and dashed purple lines display normalized EMG variations, respectively for 'stiff' and 'soft' spring configurations. Compared to 'soft' configuration, all participants had to exert greater force in 'stiff' configuration, as EMG measurements indicate greater levels for this case.

RMS (Root Mean Square) values of EMG variations were calculated for all participants, considering 'stiff' and 'soft' spring configurations. Mean and SD (Standard Deviation) values for 'stiff' and 'soft' were $(0.26 \pm 0.08)$ and $(0.03 \pm 0.01)$, respectively. 


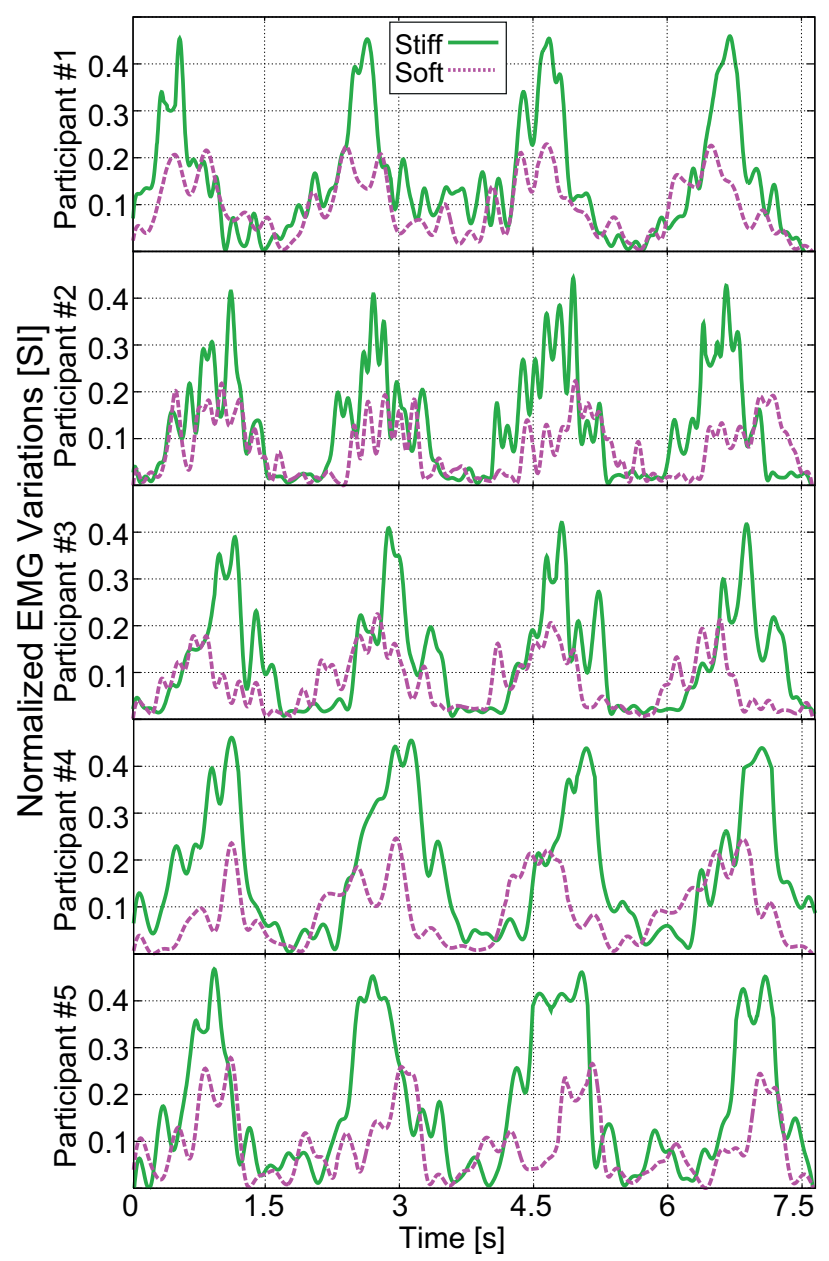

Fig. 10. Normalized EMG variations in the patient-active rehabilitation task.

In Fig. 11, human-wearer torque estimation is plotted for Participant \#3's elbow joint, both with 'stiff' and 'soft' spring configurations (solid cyan lines). Similar results were obtained for the shoulder joint. In order to confirm this estimation, we acquired the same information via sensory measurement (dashed purple). Both estimation data and actual measurement data appear to output similar variations, in which the estimation error rate does not exceed 5\%. Furthermore, Participant \#3 exerted greater torque while the virtual spring coefficient was set to 'stiff'. In comparison, user torque is $44 \%$ less in peak-to-peak values for the 'soft' spring configuration. Its RMS value also shows a $35 \%$ decrease. Similar results were obtained for all other participants.

\section{B. Patient Passive Rehabilitation Results}

Joint tracking performance is depicted in Fig. 12. Dashed purple and solid green lines respectively indicate elbow position errors while the 'proposed' scheme and 'PID' controller were applied. In addition, peak-to-peak error decreases are indicated.

Regardless of the human-wearer, joint position error never exceeded 0.25 [Deg] while the proposed scheme was implemented. Compared to conventional 'PID' controller, the elbow joint tracking error exhibits decreases, ranging from $86 \%$ to $93 \%$. Similar performance was observed for shoulder joint tracking; compared to conventional 'PID' controller, the shoulder joint tracking error shows decreases, ranging from $85 \%$ to $91 \%$.

Furthermore, peak-to-peak values of joint tracking error variations were calculated for all participants, considering the 'PID' and the

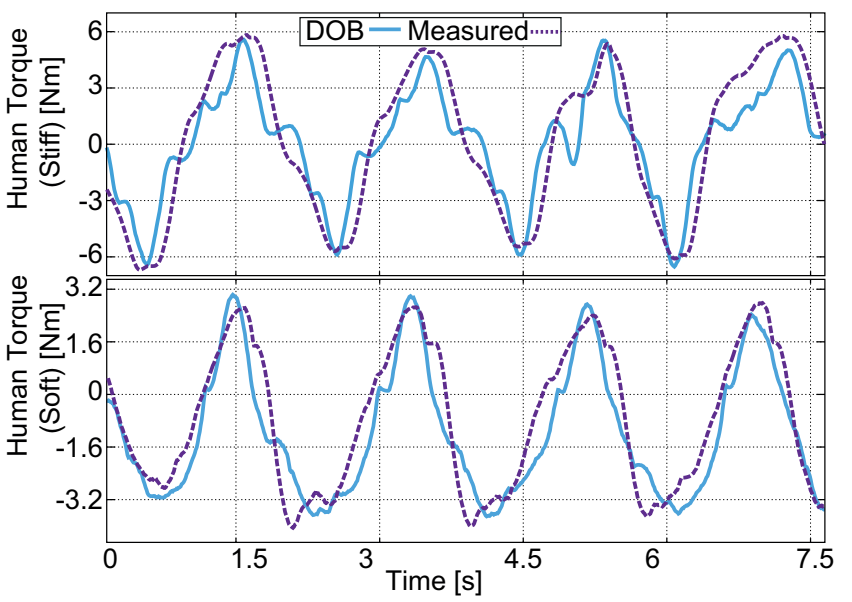

Fig. 11. Estimation of the elbow joint torque exerted by Participant \#3, during patient-active rehabilitation tests.

'proposed' controllers. Mean and SD values for 'PID' and 'proposed' were $(0.80 \pm 0.08)$ and $(0.23 \pm 0.01)$, respectively.

\section{Power Assistance Results}

In Fig. 13, normalized EMG data are displayed for all participants. In this figure, solid green lines indicate EMG variations while the participants do not wear the exoskeleton (Case 1). Dotted blue lines display EMG variations while participants wear the exoskeleton which runs the baseline algorithm, i.e., with only gravity compensation for the robot and tip weight combined (Case 2). Dashed purple lines point out EMG variations when participants wear the exoskeleton with full compensation (Case 3).

Compared to Case 1, EMG signals show decreases in RMS values, from $85 \%$ to $72 \%$, when participants wear the exoskeleton and receive power assistance with the full compensation functionality (Case 3 ). RMS value percentage decreases for this comparison (Case 1 vs Case 3) are denoted with $\zeta$ in Fig. 13.

Compared to Case 2, we obtain decreases in muscular activities; RMS values of EMG variations decrease in a range from $25 \%$ to $17 \%$, when participants wear the exoskeleton with the full compensation functionality. RMS value percentage decreases for this comparison (Case 2 vs Case 3) are indicated with $\phi$ in Fig. 13. This benchmarking study indicates that the gravitational load appears to be the major factor for the power assistance task; however, other loads, especially frictional forces also play an important role. Therefore, the proposed full compensation method outperforms the baseline approach in this specific task.

RMS values of EMG variations were calculated for all participants, considering Case 2 and Case 3. Mean and SD values for Case 2 and Case 3 are $(0.17 \pm 0.01)$ and $(0.13 \pm 0.01)$, respectively.

\section{DISCUSSION}

The patient-active rehabilitation protocol was achieved via observer-based user torque estimation, where resistive forces were adjusted using virtual impedance. In the patient-passive rehabilitation task, the proposed controller enabled precise joint tracking with a maximum positioning error of 0.25 degrees, regardless of the human arm inertial properties. In the power assistance task, the users' muscular activities were reduced up to $85 \%$ while exercising with a 5 [kg] dumbbell. 


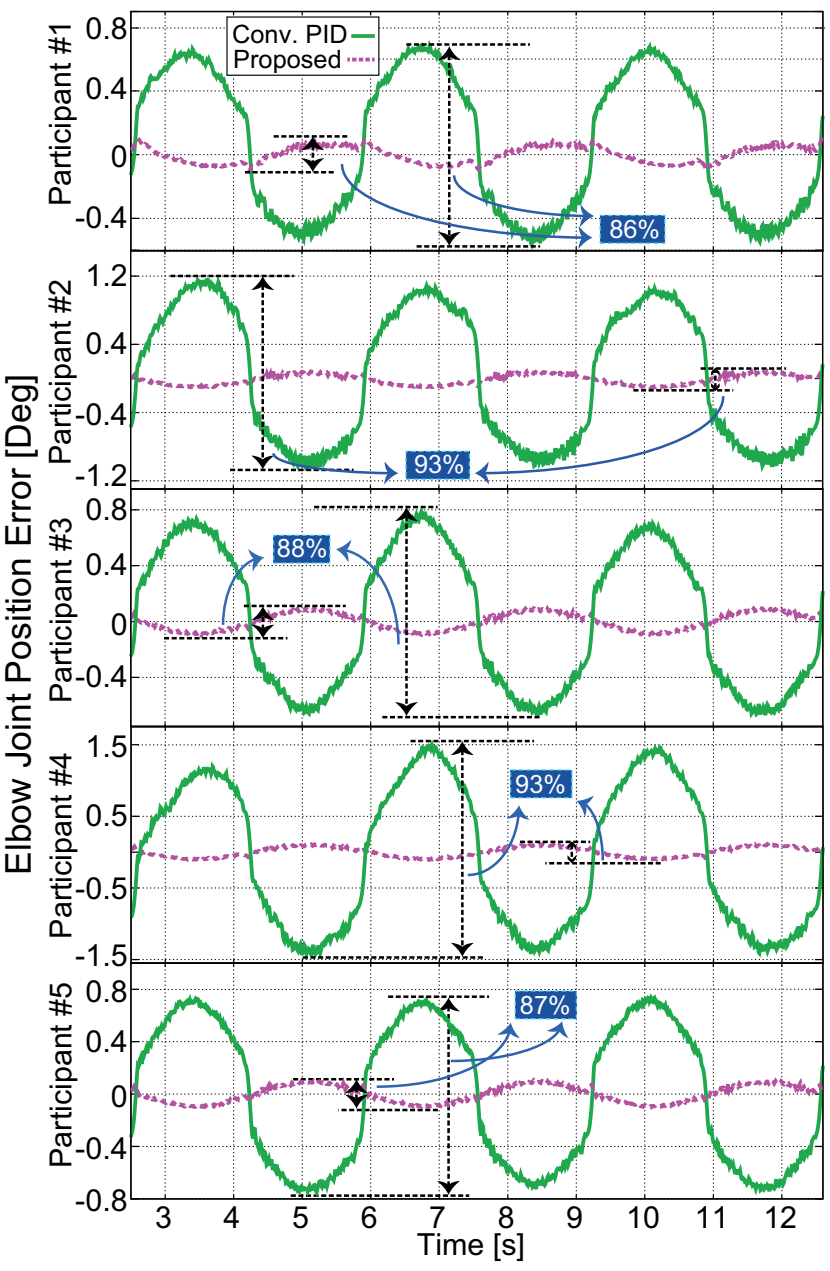

Fig. 12. Elbow joint positioning error variations for patient-passive rehabilitation tests.

The proposed system is potentially useful for patient-active and patient-passive rehabilitation tasks, which are important during muscle strengthening and rhabdomyolysis prevention treatments. In addition to rehabilitation scenarios, power assistance was demonstrated. Therefore, the proposed system may be a useful multipurpose biomedical device for rehabilitation and nursing care tasks.

The system includes only classical brushless servo motors with relatively light gears and encoders. To implement rehabilitation and power assistance tasks, we exploit off-the-shelf compensation techniques developed for robot manipulators; thus, expensive sensory and actuation units are avoided. This strategy enables us to construct a versatile system with the fewest possible elements, making it light and mobile while addressing reduced complexity.

The proposed system is subject to limitations. A single arm of the upper body exoskeleton system includes only 2 active DoFs in the shoulder and elbow, constraining the exoskeleton motion within a workspace that spans over these joints through flexion/extension. Since the main focus here is to offer a light and wearable system with reduced complexity, the number of active DoFs is limited. Therefore, the target tasks are inevitably limited with shoulder-elbow rehabilitation and power assistance scenarios along the flexion/extension axis.

Another important factor in wearable exoskeleton design is to ensure seamless connections between the exoskeleton and human limbs. This problem is addressed in several studies. For instance, Jarrasse and Morel provided formal proofs for minimizing hyper- staticity while connecting two mechanisms with different kinematic chains [23]. Ergin and Patoglu proposed and implemented a novel joint design with self-aligning capabilities [24]. In the first TTI-Exo prototype, exoskeleton link lengths are designed to be adjustable, so as to partially align human and exoskeleton joints in an attempt to prevent uncontrolled forces caused by hyperstaticity.

The proposed control framework's performance strictly depends on the robot and actuator model parameters. While robot link parameters could be obtained from CAD data with sufficient precision, one may refer to [25] to obtain a good estimation, if accurate CAD data are not accessible. A successfully implemented gravity compensation demonstration within the different configurations of workspace would provide a cross-validation for individual link mass and CoM position values as well. Furthermore, the actuator rotor inertia parameter is of importance while utilizing DOB-based estimation and control techniques. To this end, it is possible to implement system identification techniques for a disassembled actuator, to cross-check the manufacturer data. Once the robot and actuator parameters are determined to a sufficient level of precision, friction identification should be straightforward, and can be cross-validated by friction compensation demonstrations. While these procedures appear to be wellknown practices, parameter-dependency should also be addressed as a limitation.

Safety is an important concern. The exoskeleton system includes mechanical joint limits, which are designed in accordance with average human joint ranges. Furthermore, there are software and hardware controlled joint motion limitations to prevent unexpected sudden movements. Despite these efforts, the system may not be considered as inherently safe; there is a certain limitation regarding its nature [26]. Note that none of the participants reported any disturbing effect while performing given tasks with the exoskeleton system.

In the power assistance task, the tip weight to be manipulated is considered as a known load. On one hand, the necessity for this prior knowledge could be addressed as a limitation. On the other hand, it is possible to identify any unknown tip weight using the existing identification techniques [27].

Currently, the lower extremity of TTI-Exo is being employed for the paraplegia walking support task [28]. Therefore, whole-body motion control will be our next assignment.

\section{ACKNOWLEDGMENT}

This research is partially supported by Hitech Research Center, projects for private universities, from the Ministry of Education, Culture, Sports, Science and Technology, Japan. The authors thank Isil Cakir Ugurlu, Kana Kayamori, Yuta Tsuge, Hironori Oshima, Corinne Doppmann, Emmanouil Spyrakos-Papastravridis, Giuseppe Lisi, Valerio Salvucci, Arash Ajoudani, and Emre Sariyildiz for their assistance.

\section{REFERENCES}

[1] P. S. Lum, C. G. Burgar, P. C. Shor, M. Majmundar, and M. Van der Loos, "Robot-assisted movement training compared with conventional therapy techniques for the rehabilitation of upper limb motor function following stroke," in Archives of Phys. Medicine and Rehabilitation, vol. 83, no. 7, pp. 952-959, Jul. 2002.

[2] R. J. Sanchez, J. Liu, S. Rao, P. Shah, R. Smith, T. Rahman, S. C. Cramer, J. E. Bobrow, and D. J. Reinkensmeyer, "Automating arm movement training following severe stroke: Functional exercises with quantative feedback in a gravity-reduced environment," in IEEE Trans. on Neural Systems and Rehabilitation Engineering, vol. 14, no. 3, pp. 378-389, Sept. 2006.

[3] L. E. Kahn, M. L. Zygman, W. Z. Rymer, and D. J. Reinkensmeyer, "Robot-assisted reaching exercise promotes arm movement recovery in chronic hemiparetic stroke: a randomized controlled pilot study," in Journal of NeuroEngineering and Rehabilitation, vol. 3, no. 12, pp. 1-13, Jun. 2006. 


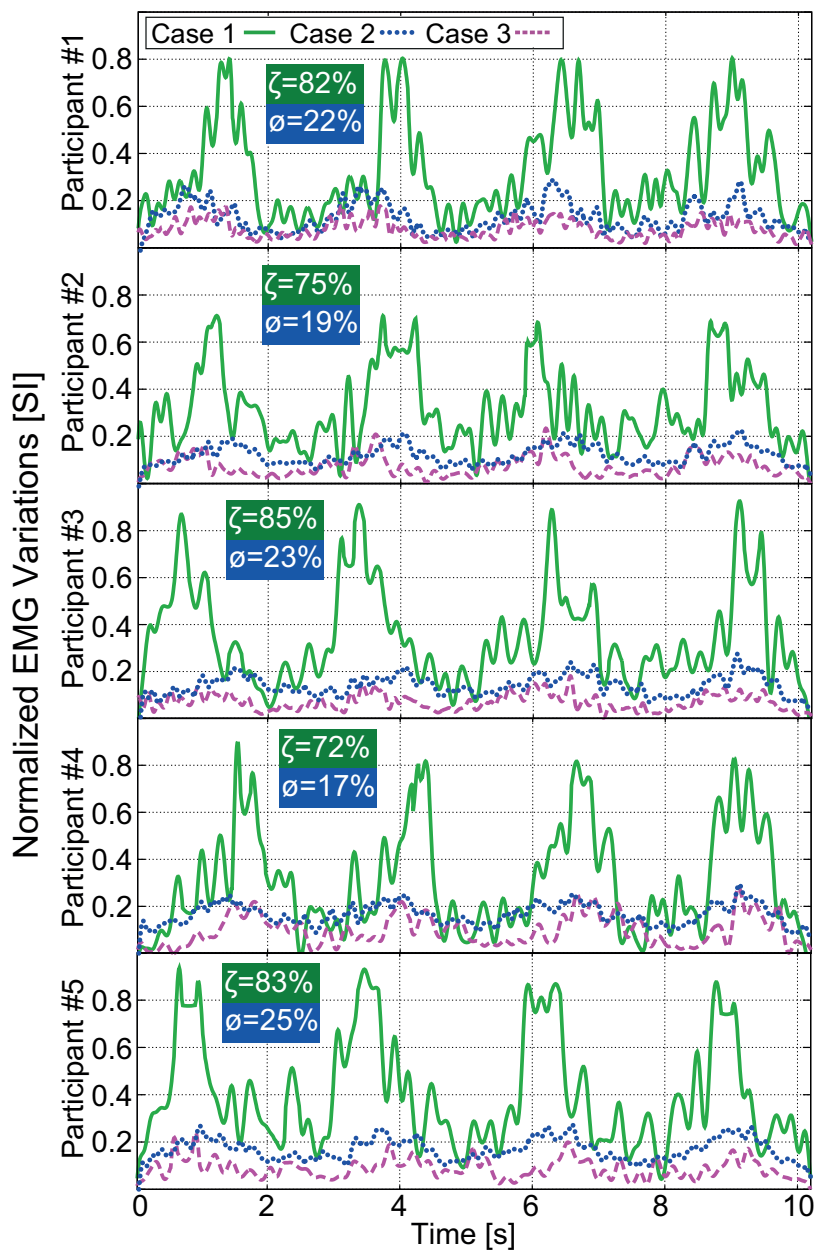

Fig. 13. Normalized EMG variations during power assistance tests.

[4] J. C. Perry, J. Rosen, and S. Burns, "Upper-limb powered exoskeleton design," in IEEE Trans. on Mechatronics, vol. 12, no. 4, pp. 408-417, Aug. 2007.

[5] T. Nef, M. Guidali, and R. Riener, "ARMin III - arm therapy exoskeleton with an ergonomic shoulder actuation," in Applied Bionics and Biomechanics, vol. 6, no. 2, pp. 127-142, Jul. 2009.

[6] H. I. Krebs, N. Hogan, M. L. Aisen, B. T. Volpe, "Robot-aided neurorehabilitation," in IEEE Trans. on Rehabilitation Engineering, vol. 6, no. 1, pp. 75-87, Mar. 1998.

[7] N. G. Tsagarakis, and D. G. Caldwell, "Improving mouse-based computer interaction in users with weak upper limb motion control during using a haptic assistive system," in IEEE Trans. on Human-Machine Systems, vol. 43, no. 2, pp. 177-187, Mar. 2013.

[8] G. Rosati, P. Gallina, and S. Masiero, "Design, implementation and clinical tests of a wire-based robot for neurorehabilitation," in IEEE Trans. on Neural Systems and Rehabilitation Engineering, vol. 15, no. 4, pp. 560-569, Dec. 2007.

[9] Y. Mao, and S. K. Agrawal, "Design of a cable-driven arm exoskeleton (CAREX) for neural rehabilitation," in Proc. IEEE Trans. on Robotics, vol. 28, no. 4, pp. 922-931, Aug. 2012.

[10] K. Ohnishi, M. Shibata, and T. Murakami, "Motion control for advanced mechatronics," in IEEE Trans on. Mechatronics, vol. 1, no. 1, pp. 56-67, Mar. 1996.

[11] T. Murakami, F. Yu, and K. Ohnishi, "Torque sensorless control in multidegree-of-freedom manipulator,' in IEEE Trans. on Industrial Electronics, vol. 40, no. 2, pp. 259-265, Apr. 1993.

[12] A. Gupta, and M. K. O'Malley, "Disturbance-observer-based force estimation for haptic feedback," in Journal of Dynamic Systems, Measurement, and Control, vol. 133, no. 1, pp. 014505-(1-4), Dec. 2010.

[13] V. Salvucci, S. Oh, and Y. Hori, "Force sensor-less power assistance control for low friction systems," in Proc. IEEE Int. Works. on Advanced Motion Control, Nagaoka, Japan, 2010, pp. 290-295.
[14] B. Ugurlu, M. Nishimura, K. Hyodo, M. Kawanishi, and T. Narikiyo, "A framework for sensorless torque estimation and control in wearable exoskeletons," in Proc. of IEEE Int. Works. on Advanced Motion Control, Sarajevo, Bosnia and Herzegovina, 2012, pp. 1-7.

[15] M. Mistry, P. Mohajerian, and S. Schaal, "An exoskeleton robot for human arm movement study," in Proc. of IEEE Conf. on Intelligent Robots and Systems, Alberta, Canada, 2005, pp. 4071-4076.

[16] D. Wade, "Rehabilitation therapy after stroke" in Lancet, vol. 354, vol. 9174, pp. 176-177, Jul. 1999.

[17] A. Gupta, and M. K. O'Malley, 'Design of a haptic arm exoskeleton for training and rehabilitation," in IEEE Trans on. Mechatronics, vol. 11, no. 3, pp. 280-289, Jun. 2006.

[18] A. Frisoli, C. Procopio, C. Chisari, I. Creatini, L. Bonfiglio, M. Bergamasco, B. Rossi, and M. C. Carboncini, "Positive effects of robotic exoskeleton training of upper limb reaching movements after stroke," in Journal of NeuroEngineering and Rehabilitation, vol. 9, no. 36, pp. 1-16, Jun. 2012.

[19] R. Vanholder, M. S. Sever, E. Erek, and N. Lameire, "Rhabdomyolysis" in Journal of the American Society of Nephrology, vol. 11, no. 8, pp. 1553-1561, Aug. 2000.

[20] D. M. Weinstein, W. J. Ciccone, M. C. Buckler, P. M. Balthrop, T. D. Busey, and J. J. Elias, "Elbow function after repair of the distal biceps brachii tendon with a two-incision approach," in Journal of Shoulder and Elbow Surgery, vol. 17, no. 1, pp. S82-S86, Jan.-Feb. 2008.

[21] D. Landin, J. Myers, M. Thompson, R. Castle, and J. Porter, "The role of the biceps brachii in shoulder elevation," in Journal of Electromyography and Kinesiology, vol. 18, no. 2, pp. 270-275, Apr. 2008.

[22] A. Ajoudani, N. G. Tsagarakis, and A. Bicchi, "Tele-impedance: teleoperation with impedance regulation using a body-machine interface," in Int. Journal of Robotics Research, vol 31, no. 13, pp. 1642-1656, Nov. 2012.

[23] N. Jarrasse, and G. Morel, "Connecting a human limb to an exoskeleton," in IEEE Trans. on Robotics, vol. 28, no. 3, pp. 697-709, Jun. 2012.

[24] M. A. Ergin, and V. Patoglu, "ASSISTON-SE: A self-aligning shoulderelbow exoskeleton," in Proc. of IEEE Int. Conf. on Robotics and Automation, St. Paul, US, 2012, pp. 2479-2485.

[25] K. Ayusawa, G. Venture, and Y. Nakamura, "Identifiability and identification of inertial parameters using the underactuated base-link dynamics for legged multibody systems," in Int. Journal of Robotics Research, vol. 33, no. 3, pp. 446-468, Mar. 2014.

[26] M. Zinn, O. Khatib, B. Roth, and J. Salisbury, 'Playing it safe [human friendly robots]," in IEEE Robotics and Automation Magazine, vol. 11, no. 2, pp. 12-21, Jun. 2004.

[27] W. Khalil, M. Gautier, and P. Lemoine, "Identification of the payload inertial parameters of industrial manipulators," in Proc. of IEEE Conf. on Robotics and Automation, Roma, Italy, 2007, pp. 4943-4948.

[28] B. Ugurlu, H. Oshima, and T. Narikiyo, "Lower body exoskeletonsupported compliant bipedal walking for paraplegics: How to reduce upper body effort?" in Proc. of IEEE Conf. on Robotics and Automation, Hong Kong, China, 2014, pp. 1354-1360.

[29] Accompanying YouTube video, including scenes from experiments. [Available Online]: http://youtu.be/3xY2mwZOP2o (Last accessed 12 June 2014) 